\title{
ESTRUTURA E PRODUÇÃO DE DUAS POPULAÇÕES NATIVAS DE CASTANHEIRA-DO-BRASIL (Bertholletia excelsa O. Berg) EM RORAIMA
}

\author{
Hélio Tonini*, Patrícia da Costa**, Paulo Emílio Kaminski** \\ *Eng. Florestal, Dr., Pesquisador da Embrapa Roraima - helio@cpafrr.embrapa.br \\ **Biólogo, M.Sc., Pesquisador da Embrapa Roraima - patricia@cpafrr.embrapa.br; emilio@cpafrr.embrapa.br
}

Recebido para publicação: 08/05/2007 - Aceito para publicação: 26/06/2008

\begin{abstract}
Resumo
Este trabalho teve como objetivo estudar a estrutura populacional, a distribuição espacial, a relação altura e diâmetro e a produção de frutos e sementes de castanheira-do-brasil em duas populações nativas localizadas no sul do estado de Roraima. Foram instaladas duas parcelas permanentes de 300 x $300 \mathrm{~m}$, onde todos os indivíduos com DAP (diâmetro tomado a $1,30 \mathrm{~m}$ do solo) igual ou superior a $10 \mathrm{~cm}$ foram identificados, mapeados e medidos. Em cada árvore foram avaliados o clima de luz, a forma da copa e a presença de cipós. Para identificar o padrão de distribuição espacial, utilizou-se a razão variância média e o índice de Morisita. A relação altura e diâmetro foi obtida por análise de regressão, sendo testados 6 modelos. Os dados de produção foram obtidos pela contagem dos frutos e pesagem das amêndoas de 150 árvores durante o ano de 2006. Como resultado, observou-se grande predominância de indivíduos adultos $(\mathrm{DAP} \geq 50 \mathrm{~cm}$ ), representando em média $69 \%$ do número total de árvores. Os indivíduos jovens $(\mathrm{DAP}<50 \mathrm{~cm})$ corresponderam a $31 \%$. A análise da distribuição espacial indicou que os indivíduos adultos apresentaram distribuição regular ou aleatória, e os jovens tendência ao agrupamento. O modelo $\ln h=b_{0}+b_{1} 1 / d$ apresentou melhor ajuste para descrever a relação altura-diâmetro, devendo ser ajustado separadamente por local. O número médio de frutos produzidos foi de 23, com média de $4,07 \mathrm{~kg}$ de sementes por árvore.

Palavras-chave: Amazônia; produtos florestais não-madeireiros; distribuição espacial; relação hipsométrica.
\end{abstract}

\begin{abstract}
Yield and structure of two Brazil nut (Bertholletia excelsa O. Berg) native populations in Roraima. This work was carried out with the objectives of studying the population structure, spatial distribution, height -diameter curve and the fruits and seeds yield of Brazil nut in two native populations in the south of the state of Roraima, Brazil. Two permanent sample plots of $300 \times 300 \mathrm{~m}$ were located and all the individuals with DBH (diameter at breast height) $\geq 10 \mathrm{~cm}$, were identified, mapped and measured. In each tree were appraised the light climate, crown form and the lianas load. To identify the spatial distribution pattern was used the medium variance/average rate and the Morisita's Index. The height - diameter relationship was obtained by regression analysis, being tested 6 models. The yield data were obtained by the count of the fruits, and weight of seeds of 150 trees during the year of 2006. As results, great predominance of adult individuals was observed (DBH $\geq 50$ $\mathrm{cm}$ ), representing $69 \%$ of the total tree number. Young individuals $(\mathrm{DBH}<50 \mathrm{~cm}$ ) corresponded to $31 \%$. The space distribution analysis showed that adult individuals presented random distribution and the youngs tendency of grouping. The model $\ln h=b_{0}+b_{1} 1 / d$ presented the best fit to describe the height

- diameter relationship should be fitted separately by place. The medium number of produced fruits was of 23 with a medium of $4,07 \mathrm{~kg}$ of seeds by tree.

Keywords: Non timber forest products; Amazon; spatial distribution; height-diameter curve.
\end{abstract}

\section{INTRODUÇ̃̃̃O}

A Bertholletia excelsa foi originalmente descrita, em 1807, por Humboldt e Bonpland. No entanto, foi Poiteau, em 1825, o primeiro a dar à Lecythidaceae o status de família, removendo os gêneros 
Bertholletia, Couratari, Couroupita e Gustavia da familia Myrtaceae, em que eram tradicionalmente classificados (MORI; PRANCE, 1990).

Trata-se de uma árvore semidecídua, heliófila, característica da mata alta de terra firme, sendo planta "social", ocorrendo em determinados locais com grande freqüência (LORENZI, 2000).

A castanheira-do-brasil desenvolve-se bem em regiões de clima quente e úmido, sendo que as maiores concentrações da espécie ocorrem em regiões onde predominam os tipos climáticos tropicais chuvosos, com a ocorrência de períodos de estiagem definidos (MÜLLER et al., 1995).

As florestas com a presença de castanheiras cobrem uma superfície de aproximadamente 325 milhões de hectares (STOIAN, 2004), abrangendo a Venezuela, a Colômbia, o Peru, a Bolívia e as Guianas. No entanto, as formações mais compactas ocorrem no Brasil (LORENZI, 2000).

Na Amazônia brasileira, as áreas produtoras de castanheira-do-brasil encontram-se sob os climas Ami e Awi, segundo a classificação de Koepen. Apresentam temperaturas médias anuais que variam entre 24,3 e $27,2{ }^{\circ} \mathrm{C}$, com valores máximos de 30,6 a 32, $6{ }^{\circ} \mathrm{C}$ e mínimos de 19,2 a $23,4{ }^{\circ} \mathrm{C}$. As médias anuais de precipitação variam entre 1400 e $2800 \mathrm{~mm}$, e a umidade relativa entre 79\% e 86\% (DINIZ e BASTOS, 1974).

As populações de castanheira-do-brasil estão situadas em solos argilosos ou argilo-arenosos de textura média a pesada (MÜLLER et al., 1995). Trata-se de uma espécie que se estabelece melhor em locais mais secos, sendo característica de mata de terra firme não-inundável (ARAÚJO et al., 2001). No leste da Amazônia, ocorre em oxissolos e ultissolos, pobres em nutrientes, porém bem estruturados e drenados, não sendo encontrada em solos excessivamente compactados (CLAY et al., 2000).

A castanheira depende da atividade dos polinizadores para assegurar a produção de frutos. Os principais polinizadores são abelhas médias e grandes, principalmente as espécies Xylocopa frontalis, Xylocopa aurulenta, Epicharis rustica, Epicaris affinis, Centris similis, Eulaema nigrita, Eulaema cingulata, Bombus brevivillus e Bombus transversalis (MAUÉS, 2002).

As sementes são disseminadas por roedores, principalmente a cutia. Algumas sementes são consumidas imediatamente, enquanto outras são armazenadas para posterior consumo ou abandonadas em outras áreas, onde germinam. Segundo Peres e Baider (1997), as sementes são dispersas a até 25 metros da árvore adulta e são enterradas individualmente a uma profundidade de $1 \mathrm{a} 3 \mathrm{~cm}$.

Em florestas naturais, a densidade varia consideravelmente, ocorrendo agrupamentos de 50 a 100 indivíduos, com 9 a 26 árvores adultas por hectare (MORI; PRANCE, 1990).

A dinâmica das populações de castanheiras em bosque primário tem sido uma questão controversa, uma vez que indivíduos jovens pertencentes às classes diamétricas menores, geralmente, são raros na floresta. Os indivíduos dessa espécie são exigentes de luz, podendo-se desenvolver em clareiras relativamente grandes. A predominância de indivíduos de grande porte indica que a espécie é dependente de clareiras (PEREIRA, 1994).

Por ser abundante na região amazônica, ser colhida quase que exclusivamente em florestas naturais, poder ser explorada por diversas comunidades no curto prazo e com baixo custo, apresentar sólida demanda de mercado e propiciar coleta com baixo impacto ambiental, a castanheira-do-brasil pode ser considerada como espécie-chave para a conservação e o desenvolvimento. Estudos recentes, como os desenvolvidos por Myers et al. (2000), Zuidema (2003), Wadt et al. (2005) e Kainer et al. (2006), têm procurado compreender a ecologia e os fatores que influenciam a produção de frutos e sementes em populações nativas de castanheira-do-brasil, enfocando a sustentabilidade e o aumento da produtividade.

Sendo assim, este trabalho teve como objetivo estudar a estrutura populacional, a distribuição espacial, a relação altura-diâmetro e a produção da castanheira-do-brasil em duas populações nativas localizadas no sul do estado de Roraima.

\section{MATERIAL E MÉTODOS}

\section{Características dos locais de coleta dos dados}

Os dados foram coletados em florestas com ocorrência natural de castanheira-do-brasil no sul do estado de Roraima, nos municípios de São João da Baliza (parcela 1), localizado nas coordenadas $00^{0} 57^{\prime} 02$ " de latitude Norte e 59 $54^{\prime} 41^{\prime \prime}$ de longitude Oeste, distante $313 \mathrm{~km}$ da capital (Boa Vista), e de Caracarai (parcela 2), nas coordenadas $01^{0} 48^{\prime} 58^{\prime \prime}$ de latitude Norte e $61^{0} 07^{\prime} 41^{\prime}$ ' de longitude Oeste, distante $135 \mathrm{~km}$ da capital (Figura 1). 


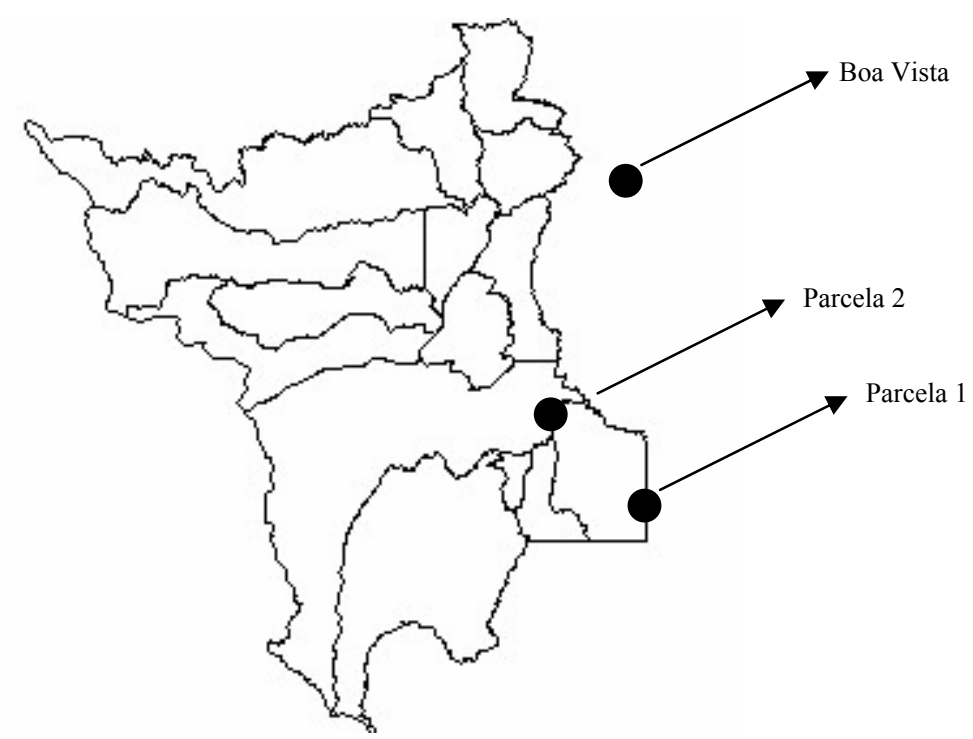

Figura 1. Localização das parcelas permanentes no estado de Roraima.

Figure 1. Permanent sample plots location in Roraima State.

A área em estudo apresenta relevo plano a ondulado, com a vegetação predominante considerada como Floresta Tropical Úmida (BRASIL, 1975). Em São João da Baliza (parcela 1), a vegetação é do tipo Floresta Ombrófila Densa, e em Caracarai (parcela 2), caracteriza-se como Floresta Tropical aberta com palmeiras.

O clima na região é classificado como Ami (tropical chuvoso com pequeno período de seca), com precipitação média anual entre 1700-2000 mm. O período chuvoso ocorre com maior freqüência de abril a agosto, com totais mensais superiores a $100 \mathrm{~mm}$. A partir de setembro ocorre uma sensível redução, com período caracteristicamente seco ocorrendo mais freqüentemente de novembro a março. A temperatura média anual é de $27{ }^{\circ} \mathrm{C}$ (FEMACT, 1993).

\section{Coleta dos dados e análise estatística}

Para a realização deste estudo, foram instaladas duas parcelas permanentes de 300 x 300 m (9 ha), subdivididas em 144 sub-amostras de $25 \times 25 \mathrm{~m}$, das quais todos os indivíduos com DAP (diâmetro tomado a $1,30 \mathrm{~m}$ do solo) igual ou superior a $10 \mathrm{~cm}$ foram identificados, mapeados e medidos. Os diâmetros foram obtidos a partir da medição da circunferência das árvores com fita métrica, e a altura total e as coordenadas ( $\mathrm{X}$ e $\mathrm{Y}$ ) com o vertex.

Em cada árvore, foram avaliados o clima de luz, a forma da copa e a presença de cipós. O clima de luz e a forma da copa foram obtidos utilizando-se o Índice de Dawkins modificado citado em Synnott (1979). A presença de cipós na copa foi obtida adotando-se os critérios empregados por Wadt et al. (2005) e Kainer et al. (2006).

Para identificar o padrão de distribuição espacial, utilizou-se a razão variância média $(\mathrm{R})$ e o Índice de Morisita (I), obtidos por:

$$
\begin{gathered}
R=\frac{s^{2}}{x} \\
I=n \frac{\sum x^{2}-N}{N(N-1)}
\end{gathered}
$$

$\mathrm{S}^{2}=$ variância da freqüência; $\mathrm{x}=$ média da freqüência; $\mathrm{n}=$ número total de parcelas; $\sum \mathrm{x}^{2}=$ soma do quadrado do número de indivíduos por parcela; $\mathrm{N}=$ número de indivíduos encontrados em todas as parcelas. 
A significância dos valores calculados para $\mathrm{R}$ e I foi obtida mediante o teste do qui-quadrado, para gl $(n-1=143)$ e um nível de significância igual a 0,05 . Valores de $\mathrm{R}$ e I menores do que 1 indicam a inexistência de agrupamento, iguais a 1 indicam distribuição regular e maiores do que 1 distribuição agregada (Krebs, 1998).

A relação altura-diâmetro foi obtida por análise de regressão, sendo testados 6 modelos (Tabela 1). A seleção da melhor equação foi realizada observando-se os critérios do coeficiente de determinação ajustado ( $\mathrm{R}^{2}$ aj), o erro padrão de estimativa em porcentagem (Syx\%), o Índice de Furnival (para as equações logarítmicas) e a análise gráfica da distribuição dos resíduos em porcentagem.

Para avaliar diferenças no padrão de crescimento em altura entre diferentes locais (parcelas), após a seleção da melhor equação de relação hipsométrica, utilizou-se o teste de identidade de modelos proposto por Graybill (1976) para avaliar a necessidade de ajuste separado por parcela, uma vez que foram alocadas em diferentes locais e tipos florísticos.

$\mathrm{O}$ teste de identidade permite observar por meio do teste $\mathrm{F}$ a significância da diferença entre $\mathrm{o}$ total das somas dos quadrados das regressões ajustadas para cada área de estudo isoladamente e a soma do quadrado da regressão ajustada para o conjunto dos dados. Nesse caso, as hipóteses foram definidas da seguinte forma:

$\mathrm{H}_{0}=$ Um mesmo modelo pode ser ajustado para a castanheira-do-brasil independentemente do local.

$\mathrm{H}_{1}=$ devem ser ajustados modelos independentes por local.

A não-rejeição da hipótese $\mathrm{H}_{0}$ permite concluir que, em certo nível de significância, as equações são idênticas. Neste trabalho, considerou-se um nível de significância de $5 \%$ de probabilidade.

Tabela 1. Modelos de relação hipsométrica ajustados.

Table1. Heigh-diameter fited models.

\begin{tabular}{lc}
\hline Número & Equação \\
\hline 1 & $h=b_{o}+b_{1} d$ \\
2 & $\ln h=b_{0}+b_{1} 1 / d$ \\
3 & $\ln h=b_{o}+b_{1} d$ \\
4 & $h=b_{o}+b_{1} d+b_{2} d^{2}$ \\
5 & $h=b_{o}+b_{1} d+b_{2} d^{2}+b_{3} d^{3}$ \\
6 & $h=b_{0}+b_{1} 1 / d$ \\
\hline $\mathrm{h}=$ altura total $(\mathrm{m}) ; \mathrm{d}=$ diâmetro tomado a $1,30 \mathrm{~m}$ do solo; $\mathrm{b}_{\mathrm{j}}=$ coeficientes dos modelos.
\end{tabular}

Para a classificação dos indivíduos em adultos e jovens adotou-se o critério utilizado por Wadt et al. (2005), baseado no estado reprodutivo. Nesse caso, foram considerados jovens, indivíduos com DAP menor do que $50 \mathrm{~cm}$.

Os dados de produção foram obtidos pela contagem dos frutos e pesagem das amêndoas em todas as árvores dentro das parcelas permanentes. As pesagens foram feitas com balança de gancho digital com precisão de $50 \mathrm{~g}$, sendo monitoradas 150 árvores durante o ano de 2006. Para avaliar a relação entre a produção de amêndoas e o clima de luz, a forma da copa e a presença de cipós, utilizou-se o coeficiente de correlação de Spearman.

\section{RESULTADOS E DISCUSSÃO}

A densidade das castanheiras variou de 3,7 a 12,9 indivíduos por hectare, o diâmetro médio apresentou maior variação entre parcelas $(65,9-74,6 \mathrm{~cm})$ do que a altura $(39,7-40,3)$, e a área basal das castanheiras variou de 1,68 a $6,9 \mathrm{~m}^{2} \mathrm{ha}^{-1}$ (Tabela 2). A maior variação de diâmetro em relação à altura também foi observada por Salomão (1991), ao constatar que, proporcionalmente, a amplitude em diâmetro das castanheiras é muito maior do que a amplitude em altura, sobretudo para árvores com DAP superior a $30 \mathrm{~cm}$. O maior diâmetro observado neste estudo foi de 152,5 cm para a parcela 1 e de 178,6 para a parcela 2. Os diâmetros médios observados foram inferiores aos obtidos por Salomão (1991) em 
Marabá (131,4 cm) e Carajás (134,8 cm), no Pará, e por Zuidema e Boot (2002) em dois sítios na Bolívia $(267$ e $190 \mathrm{~cm})$.

Tabela 2. Parâmetros dendrométricos obtidos nas parcelas permanentes.

Table 2. Dendrometric parameters obtained in permanent sample plots.

\begin{tabular}{llccccc}
\hline Parcela & Local & $\mathbf{n}$ & $\mathbf{N}(\mathbf{h a})$ & $\mathbf{d ~ ( c m )}$ & $\mathbf{h}(\mathbf{m})$ & $\mathbf{G}\left(\mathbf{m}^{2} / \mathbf{h a}\right)$ \\
\hline 1 & São João da Baliza & 33 & 3,7 & 65,9 & 40,3 & 1,68 \\
2 & Caracarai & 116 & 12,9 & 74,6 & 39,7 & 6,9 \\
\hline $\mathrm{n}=$ número de árvores na parcela (9 ha); $\mathrm{N}=$ número de árvores por hectare; $\mathrm{d}=$ diâmetro médio; $\mathrm{h}=$ altura média; $\mathrm{G}=$ área basal por hectare.
\end{tabular}

As densidades observadas neste estudo estão de acordo com as obtidas por Salomão (1991), Stoian (2004), Peres e Baider (1997) e Peres et al. (2003), que relataram uma grande variação na densidade de indivíduos de castanheira-do-brasil em diferentes locais da Amazônia $\left(\begin{array}{lll}1,3 & \text { a } & 23\end{array}\right.$ indivíduos.ha ${ }^{-1}$ ). No norte da Bolívia, segundo Peña-Claros et al. (2002), as densidades variam de 1 a 5 árvores por hectare. Salomão (1991), relatou uma densidade de 1,3 e 4,2 árvores por hectare com o DAP $\geq 10 \mathrm{~cm}$, para a região de Carajás e Marabá (PA), e densidade superior a 10 árvores/ha na Floresta Nacional de Caxiuanã (PA).

No entanto, segundo Zuidema (2003) e Wadt et al. (2005), as comparações entre densidades em diferentes estudos são prejudicadas pela inexistência de padronização nos inventários, como o emprego de unidades amostrais de diferentes tamanhos e formas, diferenças no diâmetro mínimo de amostragem, e critérios de seleção do local de instalação das unidades amostrais.

A estrutura das populações nos sítios estudados apresentou um declínio no número de indivíduos nas maiores classes diamétricas (Figura 2). As maiores densidades ocorreram nas classes intermediárias, com ausência de indivíduos em algumas classes. Esse tipo de estrutura diamétrica é a mais comum para a espécie e foi também observada nos trabalhos de Viana et al. (1998), Zuidema e Boot (2002), Zuidema (2003) e Peres et al. (2003).

Para Zuidema (2003), o acúmulo de indivíduos em certas classes diamétricas pode ser resultante do cultivo passado feito pelas populações indígenas. Nesse caso, as árvores nessas classes diamétricas teriam, aproximadamente, a mesma idade. Outra explicação refere-se às características da regeneração natural da espécie, que requer a ocorrência de grandes distúrbios, de freqüência bastante esporádica, formando agrupamentos de árvores de mesmo tamanho e idade.

Corroboram essas teorias os estudos de Pereira (1994), ao observar que os índios Kokama, do Amazonas, aplicam práticas de manejo dirigidas à fase de regeneração natural da espécie, fazendo com que os indivíduos jovens se beneficiem das clareiras dos roçados abertos em meio à vegetação natural, assim como os estudos de Myers et al. (2000), ao observarem ausência de mudas de castanheira-do-brasil sob dossel fechado e em pequenas clareiras $\left(>95 \mathrm{~m}^{2}\right)$.

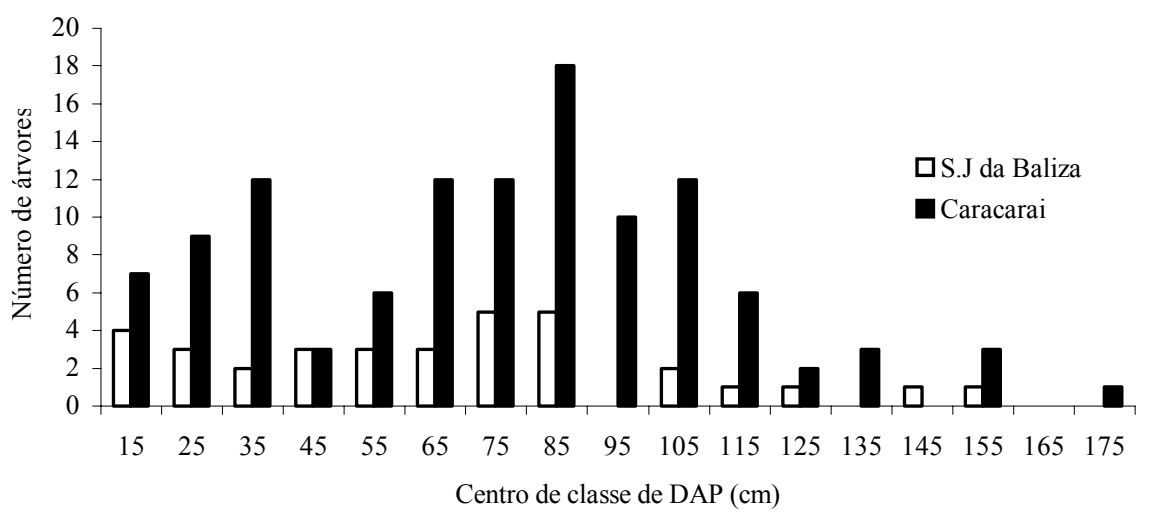

Figura 2. Estrutura das populações de castanheira-do-brasil estudadas.

Figure 2. Brazil nut population structures.

FLORESTA, Curitiba, PR, v. 38, n. 3, jul./set. 2008. 
Para Peres et al. ( 2003), o tamanho das árvores e a sua estrutura são afetadas pelo histórico de colheita. Áreas sob coleta constante são caracterizadas pela predominância de poucas árvores e poucos ou até mesmo nenhum indivíduo jovem. Indivíduos jovens são mais comuns em florestas não exploradas ou com baixa intensidade de coleta. Segundo os autores, em florestas não exploradas o número de árvores juvenis varia de 31 a 76\%, e em florestas altamente exploradas, de 0,7 a 1,6\%.

Neste estudo, foi observada grande predominância de indivíduos adultos (DAP $\geq 50 \mathrm{~cm}$ ), representando $64,7 \%$ na parcela 1 e $73,3 \%$ na parcela 2 , com uma média de $69 \%$. Os indivíduos jovens (DAP $<50 \mathrm{~cm}$ ), em média, corresponderam a $31 \%$, sendo $35,3 \%$ na parcela 1 e $26,7 \%$ na parcela 2 . Esses valores são inferiores aos observados por Viana et al. (1998) em Xapuri (AC), onde a densidade de plantas jovens (DAP $<40 \mathrm{~cm}$ ) foi quase duas vezes maior do que a de adultos, e superiores aos obtidos por Wadt (2005) na RESEX Chico Mendes (AC), que observou um número de indivíduos jovens (DAP < $50 \mathrm{~cm})$ de $23 \%$.

A porcentagem de indivíduos jovens observada neste estudo sugere que a intensidade de coleta praticada no passado foi leve. Atualmente, essas áreas localizam-se em projetos de assentamento, e pela facilidade de acesso, sofrem maior pressão de coleta.

Por outro lado, a baixa densidade de indivíduos jovens observada em estudos como os de Peres et al. (2003) pode ter sido decorrente de amostragem insuficiente para uma espécie com distribuição em mosaico (VIANA et al., 1998), ou do fato de que, devido ao comportamento heliófilo da espécie, nessas áreas existia um pequeno número de clareiras naturais. Por ser uma espécie demandante por luz, a castanheira-do-brasil regenera-se com maior sucesso em clareiras ou em florestas secundárias, e as mudas não sobrevivem por longos períodos sob dossel fechado (SALOMÃO, 1991; MYERS et al., 2000).

\section{Distribuição espacial}

Os valores calculados para a razão variância média $(\mathrm{R})$ e o índice de Morisita (I) e a significância dos valores obtidos em relação ao valor fixado para um padrão aleatório podem ser observados na tabela 3. A localização das árvores nas parcelas é apresentada na figura 3.

Tabela 3. Valores para os índices Razão de Variância média (R) e Morisita (I) para os locais estudados. Table 3. Variance mean ratio and Morisita indices for studied areas.

\begin{tabular}{lcccc}
\hline Parcela & Estágio & R & I & Prob $\chi^{\mathbf{2}}$ \\
\hline 1 & Todos & 1,14 & 1,67 & 0,12 \\
1 & Jovens & 1,26 & 4,47 & 0,02 \\
1 & Adultos & 0,86 & 0,04 & 0,88 \\
2 & Todos & 0,98 & 0,98 & 0,56 \\
2 & Jovens & 1,16 & 1,77 & 0,09 \\
2 & Adultos & 0,88 & 0,82 & 0,83 \\
\hline
\end{tabular}

A não-significância para os valores de $\mathrm{R}$ e I obtidos para toda a população e para os indivíduos adultos indicou a existência de uma distribuição regular ou aleatória. Os indivíduos jovens apresentaram tendência ao agrupamento, o que concorda com Wadt et al. (2005), que encontraram tendência ao padrão aleatório para toda a população em uma área (colocação) de 50 ha, e maior tendência ao agrupamento para as árvores juvenis.

Araújo et al. (2001), com o emprego de R e I, observaram um padrão de distribuição agrupado para árvores de castanheira-do-brasil com DAP $\geq 30 \mathrm{~cm}$ avaliadas em 158 unidades amostrais distribuídas de forma sistemática na Floresta Nacional de Carajás (PA). No entanto, esse padrão espacial pode ter sido ocasionado pela ausência da espécie em parcelas com maior umidade do solo, uma vez que o levantamento foi realizado em torno de uma barragem.

Para Wadt et al. (2005), padrões de distribuição espacial mais agrupados ou dispersos podem ser explicados, ao menos parcialmente, pelas diferenças nos tipos florestais nos quais a castanheira-do-brasil ocorre naturalmente.

\section{Relação hipsométrica}

O ajuste para os modelos testados pode ser observado na tabela 4. Pela análise do coeficiente de determinação ajustado e das medidas de dispersão, observou-se melhor ajuste com o emprego da equação 2, cuja distribuição dos resíduos pode ser observada na figura 4. 


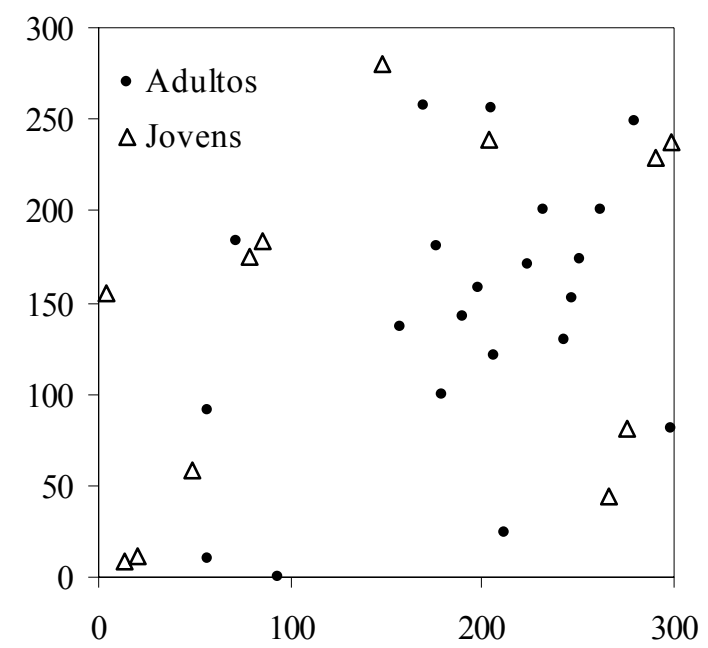

B

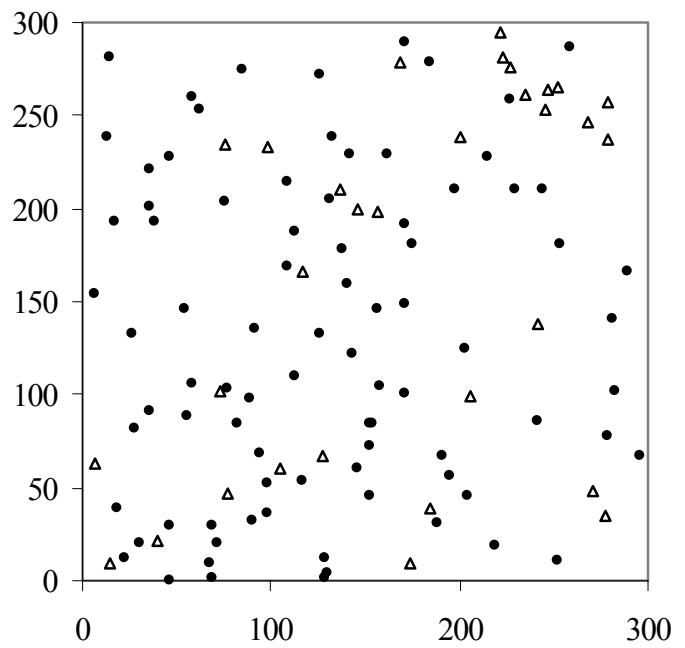

Figura 3. Distribuição espacial para árvores de castanheira-do-brasil em S. J. da Baliza (A) e Caracarai (B). Figure 3. Spatial distribution for Brazil nut trees in S. J. da Baliza (A) e Caracarai (B).

Tabela 4. Coeficientes e estatísticas de ajuste para os modelos de relação hipsométrica testados. Table 4. Coefficients and statistics of fitting for the height-diameter tested models.

\begin{tabular}{lccccccc}
\hline \multirow{2}{*}{ Número } & \multicolumn{4}{c}{ Coeficientes } & \multicolumn{3}{c}{ Estatísticas } \\
\cline { 2 - 8 } & $\mathbf{b}_{\mathbf{0}}$ & $\mathbf{b}_{\mathbf{1}}$ & $\mathbf{b}_{\mathbf{2}}$ & $\mathbf{b}_{\mathbf{3}}$ & $\mathbf{R}^{\mathbf{2}} \mathbf{\mathbf { j }}$ & $\mathbf{S y \mathbf { \% }}$ & $\mathbf{I F}$ \\
\hline 1 & 25,901 & 0,187 & & & 0,54 & 5,9 & \\
2 & 3,928 & $-14,536$ & & & 0,70 & & 3,9 \\
3 & 3,264 & 0,005 & & & 0,54 & & 3,6 \\
4 & 17,132 & 0,462 & $-0,001$ & & 0,64 & 13,0 & \\
5 & 13,187 & 0,671 & $-0,005$ & $1,08 * 10^{-5}$ & & 12,9 & \\
6 & 48,764 & $-478,393$ & & & 0,61 & 13,6 & \\
\hline
\end{tabular}

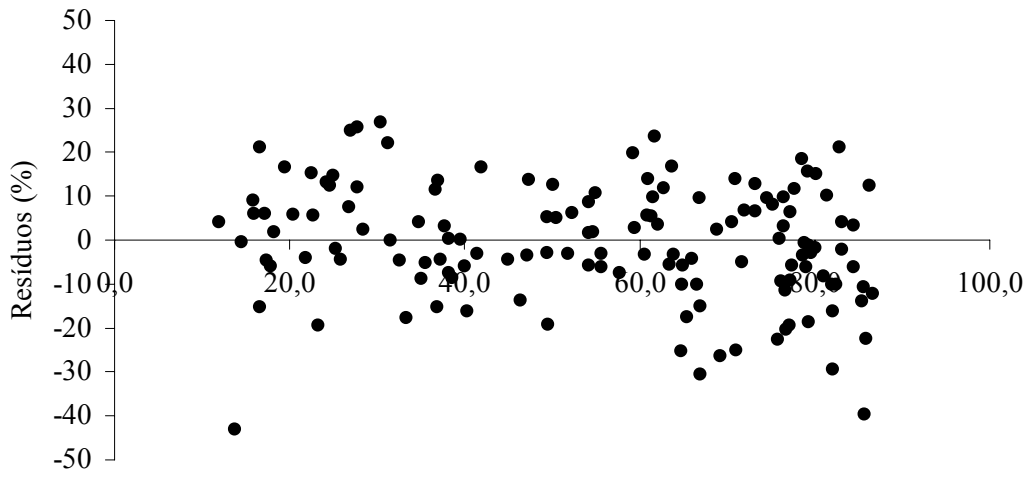

$\operatorname{DAP}(\mathrm{cm})$

Figura 4. Distribuição percentual dos resíduos em função do DAP para o modelo 2.

Figure 4. Distribution of residuals in percentage for model 2. 
O teste de identidade de modelos indicou ser inadequado o agrupamento dos dados, sendo necessário o ajuste em separado (Tabela 5), pois o valor do $\mathrm{F}$ calculado foi igual a 3,44, superior ao tabelado $(3,07)$ para $5 \%$ de probabilidade. A figura 5 mostra as estimativas obtidas com o ajuste da equação hipsométrica de forma separada e comprova graficamente os resultados obtidos com o teste de identidade, sendo possível observar um maior achatamento na curva de relação hipsométrica para as árvores da parcela 2. As razões para essa diferença não podem ser explicadas apenas com os dados coletados para a elaboração deste trabalho, porém, por se tratar de uma espécie dominante que somente desenvolve plenamente suas copas acima do dossel, o esperado é que sua altura esteja altamente correlacionada com a altura média da floresta, que pode variar com o tipo fisionômico.

Tabela 5. Resultado do teste de identidade de modelos para o ajuste de equação hipsométrica para a castanheira-do-brasil nas duas áreas estudadas.

Table 5. Model identity test results for height-diameter curves for Brazil nut in two studied areas.

\begin{tabular}{lccc}
\hline $\begin{array}{l}\text { QM diferença para testar } \\
\text { a hipótese }\end{array}$ & QM do resíduo & F calculado & F tabelado (5\%) \\
\hline 0,0613 & 0,0178 & 3,44 & 3,07 \\
\hline
\end{tabular}

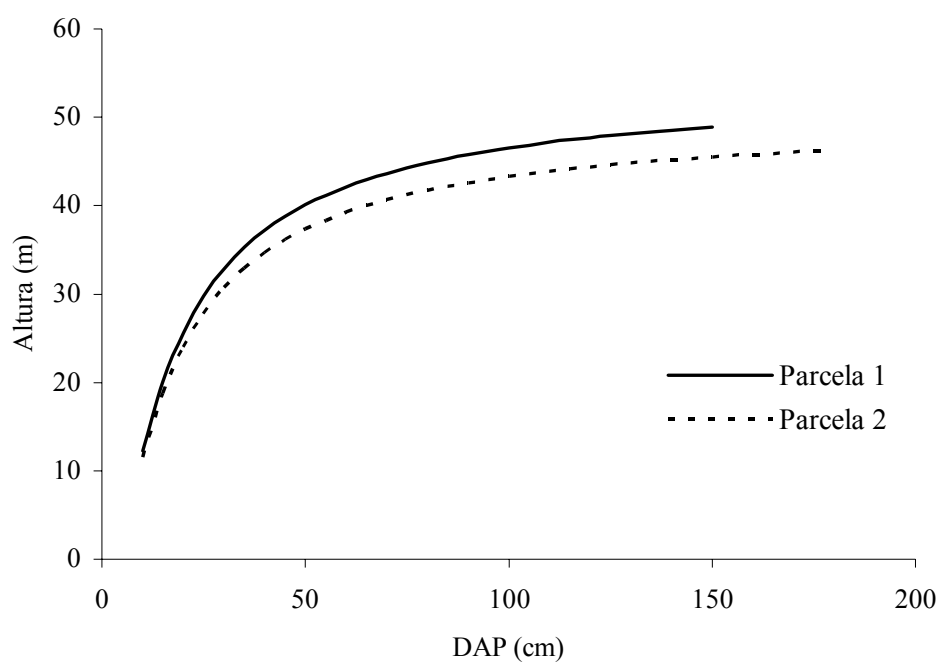

Figura 5. Relação hipsométrica para duas populações de castanheira-do-brasil em Roraima.

Figure 5. Height-diameter curves for two Brazil nut populations in Roraima.

Através da curva de relação hipsométrica, observou-se maior inclinação para árvores jovens (DAP $<50 \mathrm{~cm}$ ), o que reflete a estratégia de crescimento da espécie que busca atingir as camadas superiores do dossel rapidamente e concorda com o padrão de crescimento em altura descrito por Zuidema (2003). Para Salomão (1991), após a germinação da semente, o crescimento em altura da castanheira-do-brasil é extremamente rápido, pois a competição por luz na floresta é de vital importância para a espécie, implicando o estabelecimento ou não do indivíduo no ambiente.

\section{Análise da produção de frutos} tabela 6 .

Os dados de produção por árvore obtidos nas parcelas permanentes podem ser observados na

Na parcela 1, a produção total de sementes foi de $76,32 \mathrm{~kg}$, o que equivale a $32,7 \mathrm{~kg} / \mathrm{ha}$, produzidos por 67 árvores (63,2\%). Na parcela 2, a produção total foi de $295 \mathrm{~kg}(8,48 \mathrm{~kg} / \mathrm{ha})$ e $77,4 \%$ das árvores produziram.

Apesar da grande diferença de densidade de castanheiras entre as parcelas, não houve diferença estatística significativa entre o peso de sementes e o número de frutos por árvore entre parcelas $(\mathrm{p}=0,14$ 
e $\mathrm{p}=0,38$, respectivamente). Considerando-se toda a população, o número médio de frutos produzidos foi de 23, com uma produção média de $4,07 \mathrm{~kg}$ de sementes por árvore. Esses valores são inferiores aos observados por diferentes pesquisadores em reservas extrativistas no Acre. Viana et al. (1998) observaram uma produção média por árvore de $24 \mathrm{~kg}$, com um mínimo de $1,5 \mathrm{~kg}$ e um máximo de 105 $\mathrm{kg}$. Wadt et al. (2005) observaram uma produção média de 10,28 kg.arvore ${ }^{-1}$ em 140 árvores-amostra com DAP mínimo $\geq 10 \mathrm{~cm}$, e Kainer et al. (2006), monitorando 140 árvores durante dois anos consecutivos, obtiveram um número médio de frutos coletados de 65,5 e 72,2 e peso de sementes de 9,7 $\mathrm{kg}$ e $10,7 \mathrm{~kg}$. Homa et al. (2000) relataram média de produção de 0,46 hectolitros por árvore, o que corresponde a cerca de $23 \mathrm{~kg}$. Desconsiderando-se as árvores jovens, a produção média aumenta para $4,62 \mathrm{~kg}$ por árvore.

Tabela 6. Dados de produção por árvore obtidos nas parcelas permanentes.

Table 6. Brazil nut yield obtained in permanent sample plots.

\begin{tabular}{lcccccc}
\hline \multirow{2}{*}{ Parcela } & \multicolumn{3}{c}{ Número de frutos } & \multicolumn{3}{c}{ Peso das sementes } \\
\cline { 2 - 7 } & média & max & min & média & max & min \\
\hline 1 & 24,8 & 155 & 1 & 4,3 & 32,1 & 0,3 \\
2 & 18,6 & 80 & 1 & 2,8 & 10,65 & 0,1 \\
Média geral & 23,3 & & & 4,07 & & \\
\hline
\end{tabular}

Segundo Zuidema (2003), a maioria das árvores de castanheira-do-brasil produz poucos frutos, sendo que a maioria dos adultos produz menos de 100. Uma parte substancial da população produz de 100 a 200 frutos, e menos de $10 \%$ produzem acima de 500 . No presente estudo, o número máximo de frutos produzidos foi de 155 , sendo que $46.6 \%$ dos indivíduos produziram até 10 frutos, 35,5\% produziram entre 10 e 50 frutos, $13,3 \%$ entre 50 e 100 e apenas 4,4\% produziram mais de 100 .

$\mathrm{Na}$ análise descritiva da população estudada (Tabela 7), observa-se que, a partir de $50 \mathrm{~cm}$ de DAP, considerado como o diâmetro limite para a produção, nenhuma árvore apresentou posição da copa nas classes inferiores do dossel, ou seja, todos os indivíduos apresentaram copas dominantes ou codominantes.

Tabela 7. Caracterização de 150 árvores de castanheira-do-brasil de acordo com a classe de diâmetro, a posição e a forma e presença de cipós na copa, nas duas áreas estudadas.

Table 7. Characteristics of 150 Brazil nut trees by diameter class, crown position, crown form and lianas load in studied areas.

\begin{tabular}{|c|c|c|c|c|c|c|c|c|c|c|c|c|c|c|c|}
\hline \multirow{2}{*}{$\begin{array}{l}\text { Classe } \\
\text { diamétrica }\end{array}$} & \multirow[b]{2}{*}{$\mathbf{N}$} & \multirow[b]{2}{*}{$\mathrm{Nr} \%$} & \multicolumn{4}{|c|}{ Posição da copa (\%) } & \multicolumn{4}{|c|}{ Forma da copa (\%) } & \multicolumn{5}{|c|}{ Cipó na copa (\%) } \\
\hline & & & D & CD & I & $\mathbf{S}$ & $\mathbf{C P}$ & B & I & $\mathbf{P}$ & MP & $\mathbf{0}$ & $\leq \mathbf{2 5}$ & $25-75$ & $>75$ \\
\hline $10 \leq \mathrm{DAP}<50$ & 43 & 16,3 & 14,0 & 55,8 & 16,3 & 14,0 & 23,3 & 53,5 & 9,3 & 14,0 & 0 & 53,5 & 18,6 & 9,3 & 16,3 \\
\hline $50 \leq \mathrm{DAP}<100$ & 74 & 71,6 & 70,3 & 29,7 & 0 & 0 & 40,5 & 52,7 & 6,8 & 1,4 & 0 & 51,4 & 18,9 & 13,5 & 16,2 \\
\hline $100 \leq \mathrm{DAP}<150$ & 28 & 82,1 & 78,6 & 21,4 & 0 & 0 & 67,8 & 25,0 & 3,6 & 3,6 & 0 & 64,3 & 28,6 & 3,6 & 3,6 \\
\hline $\mathrm{DAP} \geq 150$ & 5 & 80 & 100,0 & 0,0 & 0 & 0 & 80,0 & 20,0 & 0 & 0 & 0 & 60,0 & 40,0 & 0 & 0 \\
\hline Total & 150 & 62,5 & 65,7 & 26,7 & 4,1 & 3,5 & 52,9 & 37,8 & 4,9 & 4,7 & 0 & 57,3 & 26,5 & 6,6 & 9,0 \\
\hline
\end{tabular}

$\mathrm{N}=$ número de árvores; $\mathrm{Nr} \%=$ número de árvores reprodutivas em porcentagem; $\mathrm{D}=$ dominante (recebe plena luz lateral e direta); $\mathrm{CD}=$ codominante (recebe apenas luz direta); $\mathrm{I}=$ intermediária (recebe apenas alguma luz direta ou lateral); $\mathrm{S}=$ suprimida (nenhuma luz direta); $\mathrm{CP}=$ forma da copa circular completa; $\mathrm{B}=$ forma circular, porém irregular; $\mathrm{I}=$ meia-copa; $\mathrm{P}=$ menos do que meia copa; $\mathrm{MP}=$ presença de apenas um ou menos galhos.

A grande maioria dos indivíduos apresentou forma da copa boa ou regular (92,4\%). Indivíduos com malformação da copa foram mais freqüentes nas menores classes de DAP, o que caracteriza o comportamento heliófilo da espécie.

Não foi observada a presença de cipós na copa em 57,3\% dos indivíduos. Nas maiores classes diamétricas, o número de árvores com alta infestação de cipós foi baixo, sendo o percentual de árvores com mais de $75 \%$ da copa infestada pequeno ( $9 \%$ ). No entanto, os maiores percentuais foram observados nas menores classes diamétricas, o que pode prejudicar a produção futura de sementes, pois, segundo estudos como os de Wadt et al. (2005) e Kainer et al. (2006), a presença de cipós na copa influencia negativamente a produção de frutos e sementes de castanheira-do-brasil. 
O percentual de árvores em produção aumentou com a classe de diâmetro, sendo maior na classe entre $100 \leq \mathrm{DAP}<150$, com $82,1 \%$. A produção total foi maior na classe $50 \leq \mathrm{DAP}<100$, e árvores com DAP superior ou igual a $150 \mathrm{~cm}$ obtiveram maior produção individual.

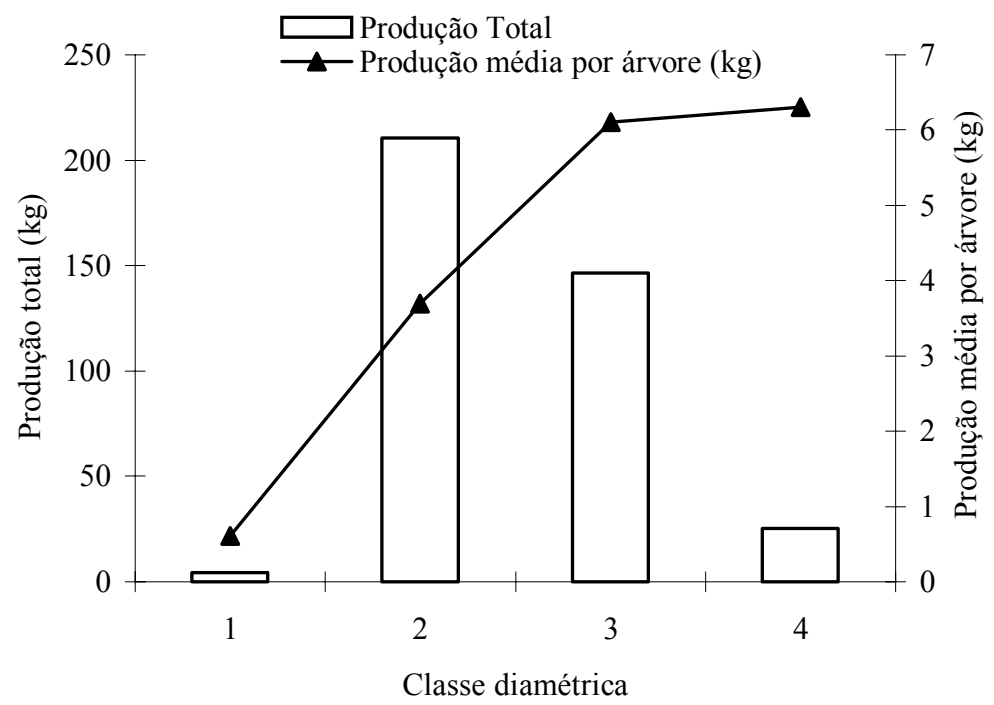

Figura 6. Produção total e individual de árvores de castanheira-do-brasil por classe diamétrica.

Figure 6. Total and individual production of Brazil nut trees by diametric class.

A correlação entre a produção de sementes $(\mathrm{kg})$ e as variáveis DAP, forma e posição da copa e infestação por cipós na copa pode ser observada na tabela 8 .

Tabela 8. Coeficiente de Correlação de Spearman entre as variáveis produção de sementes (kg), forma da copa (FC), posição da copa (PC), diâmetro à altura do peito (DAP) e presença de cipó na copa (CC).

Table 8. Spearman Coefficient of Correlation among seeds yield (kg), crown form (FC), crown position (PC), diameter at breast height (DAP) and liana load (CC).

\begin{tabular}{lccccc}
\hline & Produção & FC & PC & DAP & CC \\
\hline Produção & 1 & $-0,432^{* *}$ & $-0,363^{* *}$ & $0,476^{* *}$ & $-0,003$ \\
FC & $-0,432^{* *}$ & 1 & $0,433^{* *}$ & $-0,489^{* *}$ & 0,145 \\
PC & $-363^{* *}$ & $0,433^{* *}$ & 1 & $-0,530^{* *}$ & 0,127 \\
DAP & $0,476^{* *}$ & $-0,489^{* *}$ & $-0,530^{* *}$ & 1 & $-0,128$ \\
CC & $-0,003$ & 0,145 & 0,127 & $-0,128$ & 1 \\
\hline
\end{tabular}

** Significativo para 0,01 de probabilidade de confiança.

A produção de árvores de Castanheira-do-Brasil correlacionou-se de forma significativa com o DAP e a forma e posição da copa. Correlações significativas entre a produção de sementes e essas variáveis também foram obtidas por Viana et al. (1988), Zuidema; Boot (2002) e Wadt et al. (2005). Não foram observadas correlações significativas entre a presença de cipós na copa e a produção de sementes e a forma da copa, o que não concorda com os estudos de Wadt et al. (2005) e Kainer et al. (2006). No entanto, apesar de não ser significativo, observou-se tendência à redução no número de árvores em produção com o aumento da presença de cipós na copa (Figura 7). 
A

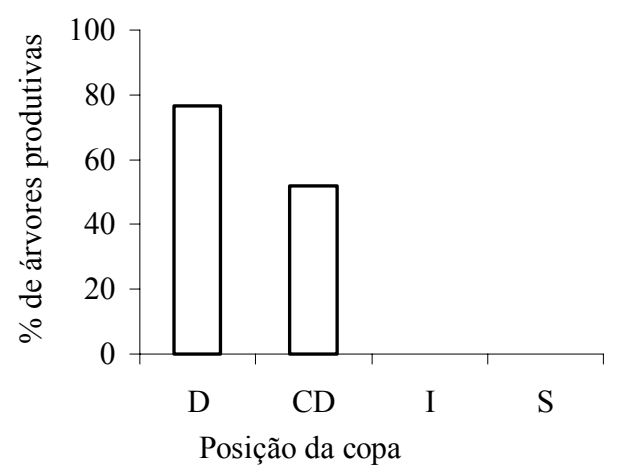

B

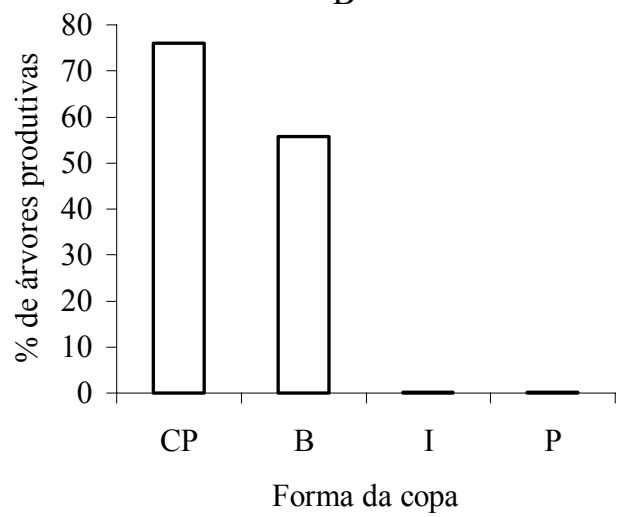



Figura 7. Relação observada entre o número de árvores em produção e a forma da copa (A), a posição da copa (B) e a presença de cipós (C).

Figure 7. Observed relationship among productive tree number and crown form (A), crown position (B) and lianas load (C).

\section{CONCLUSÕES}

Após a realização deste estudo, pode-se concluir que:

- A estrutura das populações nos sítios estudados apresentou um menor número de indivíduos nas maiores classes diamétricas, e as maiores densidades ocorreram nas classes intermediárias. Foi observada grande predominância de indivíduos adultos (DAP $\geq 50 \mathrm{~cm}$ ), representando em média $69 \%$ do número total de árvores. Os indivíduos jovens (DAP $<50 \mathrm{~cm}$ ), em média, corresponderam a 31\%.

- A análise da distribuição espacial indicou que os indivíduos adultos apresentaram distribuição regular ou aleatória. Os indivíduos jovens apresentaram tendência ao agrupamento. 
- O modelo $\ln h=b_{0}+b_{1} 1 / d$ apresentou melhor ajuste para descrever a relação altura-diâmetro de árvores nativas de castanheira-do-brasil, devendo ser ajustada separadamente por local.

- A produção de frutos e sementes pode ser considerada baixa na comparação com estudos semelhantes realizados na região amazônica. O número médio de frutos produzidos foi de 23 , com uma produção média de $4,07 \mathrm{~kg}$ de sementes por árvore.

- A produção de amêndoas correlacionou-se de forma significativa com o DAP, a forma e a posição da copa.

\section{REFERÊNCIAS}

ARAUJO, M. M.; OSAQUI, H.; MELO, R. S. Padrão de distribuição espacial de castanheira (Bertholletia excelsa H.B.K), barragem do contado, Floresta Nacional de Carajás, Pará. In: SIMPÓSIO LATINO-AMERICANO SOBRE MANEJO FLORESTAL, 2 , 2001, Santa Maria. Anais... Santa Maria: UFSM, 2001. p. 367-375.

BRASIL. Departamento Nacional da produção Mineral.Projeto RADAMBRASIL: Folha NA.20 Boa Vista e parte das folhas NA 21, Tumucumaque, N. 20 Roraima e NB 21; geologia, geomorfologia, pedologia, vegetação e uso potencial da terra. Rio de Janeiro, 1975, 428 p.

CLAY, J. W.; SAMPAIO, P. T. B.; CLEMENT, C. R. Biodiversidade amazônica: exemplos e estratégias de utilização. Manaus: INPA, 2000. 409 p.

DINIZ, T. D. A. S.; BASTOS, T. X. Contribuição ao conhecimento do clima típico da Castanha-doBrasil. Belém: IPEAN, 1974. p. 59-71 (Boletim Técnico, n. 064).

FUNDAÇÃO DO MEIO AMBIENTE CIÊNCIA E TECNOLOGIA DO ESTADO DE RORAIMA. O Brasil do hemisfério norte: diagnóstico científico e tecnológico para o desenvolvimento. Boa Vista: Ambtec, 1993, $512 \mathrm{p}$.

HOMA, A. K. O.; CARVALHO, R. A.; FERREIRA, C. A. P. As razões econômicas da destruição de um recurso natural: o caso de castanhais no sudeste paraense. In: CONGRESSO MUNDIAL DE SOCIOLOGIA RURAL, 10, 2000, Rio de Janeiro. Anais do ... Rio de Janeiro: IRSA, 2000. p. 1-15.

KAINER, K. A.; WADT, L. H. O.; GOMES-SILVA, D. A. P.; CAPANU, M. Liana loads and their association with Bertholletia excelsa fruit and nut production, diameter growth and crown attributes. Journal of Tropical Ecology, Cambridge, GB, v. 22, p. 147-154, 2006.

KREBS, C. J. Ecological methodology. New York: Harper Collins, 1998, 620 p.

LORENZI, H. Árvores brasileiras, 4. ed. São Paulo: Instituto Plantarum, 2000. v.1.

MAUÉS, M. M. Reproductive phenology and pollination of the Brazil nut tree (Bertholletia excelsa Humb. \& Bonpl.) in eastern Amazonian. In: KEVAN P; IMPERATRIZ F. (Eds.). Pollinating bees: the conservation link between agriculture and nature. Brasília, DF: Ministério do Meio Ambiente, 2002, p. 245-254.

MULleR, C. H.; FIQUEIREDO, F. J. C.; KATO, A. K.; CARVAlHO, J. E. U.; STEIN, R. L. B.; SILVA, A. B. Castanha-do-Brasil. Brasília: Embrapa SPI, 1995, 65 p. (Coleção plantar).

MORI, S. A.; PRANCE, G. T. Taxonomy, ecology and economic botany of the Brazil nut (Bertholletia excelsa, Humb \& Bonpl: Lecythidaceae). In: PRANCE, G. T.; BALICK, M. J. (Eds.). New directions in the study of plants and people: research contributions from the Institute of Economic Botany. New York: The New York Botanical Garden, 1990. v.8, p.130-150. (Advances in economic botany).

MYERS, G. P.; NEWTON, A. C.; MELGAREJO, O. The influence of canopy gap size on natural regeneration of Brazil nut (Bertholletia excelsa) in Bolivia. Forest ecology and management, Amsterdam, v. 127, p.119-128, 2000. 
PEÑA-CLAROS, M.; BOOT, R. G. A.; LORA, J. D.; ZONTA, A. Enrichment planting of Bertholletia excelsa in a secondary forest in the Bolivian Amazon: effect of cutting line width on survival, growth and crown traits. Forest ecology and management, Amsterdam, v. 161, p. 159-168, 2002.

PERES, C. A.; BAIDER, C. Seed dispersal, spatial distribution and population structure of Brazil nut trees (Bertholletia excelsa) in Southeastern Amazonia. Journal of Tropical Ecology, Cambridge, GB, v. 13, p. 595-616, 1997.

PERES, C. A.; BAIDER, C.; ZUIDEMA, P. A.; et al. Demographic threats to the sustainability of Brazil nut exploitation. Science, Washington, DC, v. 1, p. 12-14, 2003.

PEREIRA, H. S. Manejo agroflorestal da castanheira (Bertholletia excelsa, H.B.K) na Região do Lago de Tefé (AM). Revista da Universidade do Amazonas. Série Ciências Agrárias, Manaus, v. 3, n. 1, p. 1132, 1994.

SALOMÃO, R. P. Estrutura e densidade de Bertholletia excelsa H.\&B. (castanheira) nas regiões de Carajás e Marabá, estado do Pará. Boletim do Museu Paraense Emílio Goeldi. Série Botânica, Belém, v. 7, n. 1, p. 47-68, 1991

SYNNOTT, T. J. A manual of permanent sample plot procedures for tropical rainforests. Oxford: Commonwealth Forestry Institute: University of Oxford, 1979. (Tropical Forestry Papers, n.14).

STOIAN, D. Cosechando lo que cae:la economia de la castaña (Bertholletia excelsa H.B.K) em la Amazônia Boliviana. In: ALEXIADES, M.N.; SHANLEY, P. (Eds.) Productos forestales, medios de subsistencia y conservación de productos forestales no maderables. Cifor, 2004. v.3, cap.5, p. 89-116.

VIANA, V. M.; MELLO, R. A.; MORAES, L. M, et al. Ecologia e manejo de populações de castanhado-Pará em reservas extrativistas, Xapuri, Estado do Acre. In: GASCON, C.; MOUTINHO, P. (Eds.). Floresta Amazônica: dinâmica, regeneração e manejo. Manaus: INPA, 1978. 373 p.

ZUIDEMA, P. A.; BOOT, R. G. A. Demography of the Brazil nut tree (Bertholletia excelsa) in the Bolivian Amazon: Impact of seed extraction on recruitment and population dynamics. Journal of Tropical Ecology, Cambridge, GB, v. 18, p. 1-31, 2002.

ZUIDEMA, P. A. Demography and management of the Brazil nut tree (Bertholletia excelsa). Riberalta: PROMAB, 2003. 111 p. (PROMAB Scientific Series, n.6).

WADT, L. H. O.; KAINER, K. A.; GOMES-SILVA, D. A. P. Population structure and nut yield of a Bertholletia excelsa stand in Southwestern Amazonia. Forest Ecology and Management, Amsterdam, v. 211, p. 371-384, 2005. 